\title{
A GLIMM TYPE FUNCTIONAL FOR A SPECIAL JIN-XIN RELAXATION MODEL
}

\author{
Stefano BIANCHINI ${ }^{1}$ \\ SISSA-ISAS, via Beirut 2-4, 34014 Trieste, Italy
}

Manuscript received 18 January 2000

ABSTRACT. - We consider a special case of the Jin-Xin relaxation systems

$$
u_{t}+v_{x}=0, \quad v_{t}+\lambda^{2} u_{x}=(F(u)-v) / \varepsilon .
$$

We assume that the integral curves of the eigenvectors $r_{i}$ of $D F(u)$ are straight lines.

In this setting we prove that for every initial data $u, v$ with sufficiently small total variation the solution $\left(u^{\varepsilon}, v^{\varepsilon}\right)$ of $(*)$ is well defined for all $t>0$, and its total variation satisfies a uniform bound, independent of $t, \varepsilon$. Moreover, as $\varepsilon$ tends to $0^{+}$, the solutions $\left(u^{\varepsilon}, v^{\varepsilon}\right)$ converge to a unique limit $(u(t), v(t)): u(t)$ is the unique entropic solution of the corresponding hyperbolic system $u_{t}+F(u)_{x}=0$ and $v(t, x)=F(u(t, x))$ for all $t>0$, a.e. $x \in \mathbb{R}$.

The proofs rely on the introduction of a new functional for the solutions of $(*)$, corresponding to the Glimm interaction potential for the approaching waves of different families.

(C) 2001 L'Association Publications de l'Institut Henri Poincaré. Published by Elsevier B.V. All rights reserved

AMS classification: $35 \mathrm{~L} 65$

RÉSUMÉ. - Nous considérons un cas special des systèmes de relaxation

$$
u_{t}+v_{x}=0, \quad v_{t}+\lambda^{2} u_{x}=(F(u)-v) / \varepsilon .
$$

Nous supposons que les courbes intégrales des vecteurs propres $r_{i}$ de $D F(u)$ sont des droites.

Sous ces hypothèses, nous prouvons que pour chaques données initiales $u, v$ avec une variation totale suffisamment petite la solution $\left(u^{\varepsilon}, v^{\varepsilon}\right)$ de $(*)$ est bien définie pour tout $t>0$, et sa variation totale satisfait une borne uniforme, indépendante de $t, \varepsilon$. De plus, quand $\varepsilon$ tend vers $0^{+}$, les solutions $\left(u^{\varepsilon}, v^{\varepsilon}\right)$ convergent vers une unique limite $(u(t), v(t)): u(t)$ est l'unique solution entropique du système hyperbolique correspondant $u_{t}+F(u)_{x}=0$ et $v(t, x)=F(u(t, x))$ pour tout $t>0$, p.p. $x \in \mathbb{R}$. Les preuves sont basées sur l'introduction d'une nouvelle fonctionnelle pour les solutions de $(*)$, correspondant au potential d'interaction de Glimm pour les vagues approchantes des différentes familles.

(C) 2001 L'Association Publications de l'Institut Henri Poincaré. Published by Elsevier B.V. All rights reserved

\footnotetext{
E-mail address: bianchin@sissa.it (S. Bianchini).

${ }^{1}$ I thank Davide Guzzetti for his clarifying explanation on complex analysis. I also thank Prof. Alberto Bressan for his careful reading of the manuscript.
} 


\section{Introduction}

Consider the following $n \times n$ hyperbolic system of conservation laws

$$
u_{t}+F(u)_{x}=0, \quad(x, t) \in \mathbb{R} \times \mathbb{R}^{+}, u \in \mathbb{R}^{n},
$$

where $F$ is a smooth vector valued function. In [8], S. Jin and Z. Xin proposed to approximate (1.1) by the relaxation system

$$
\left\{\begin{array}{l}
u_{t}+v_{x}=0, \\
v_{t}+\Lambda u_{x}=(F(u)-v) / \varepsilon,
\end{array}\right.
$$

where $u, v \in \mathbb{R}^{n}$ and $\Lambda=\operatorname{diag}\left(\lambda_{1}^{2}, \ldots, \lambda_{n}^{2}\right)$ is a $n \times n$ diagonal matrix. As $\varepsilon$ tends to zero, at least at a formal level one obtains the equations [8]

$$
v=F(u), \quad u_{t}+F(u)_{x}=0 .
$$

The above problem is closely related to the convergence of the vanishing viscosity limit

$$
u_{t}+F(u)_{x}-\varepsilon u_{x x}=0 .
$$

In fact, for small $\varepsilon$, applying the Chapman-Enskog expansion in the relaxation system (1.2), we obtain the following approximation

$$
u_{t}+F(u)_{x}=\varepsilon\left(\left(\Lambda-(A(u))^{2}\right) u_{x}\right)_{x},
$$

where $A(u)$ is the Jacobian matrix of the flux function $F$. This relation shows that a necessary stability condition is $\Lambda-(A(u))^{2}>0$. For a survey of hyperbolic relaxation problems see [11].

In this paper we consider a simplified version of the system (1.2). More precisely we assume $\Lambda=\lambda^{2} I$, where $I$ is the $n \times n$ identity matrix, $\lambda$ is a sufficiently big constant, and $F(u)$ is a vector valued function such that its Jacobian matrix is strictly hyperbolic in an open set $\Omega \subseteq \mathbb{R}^{n}$. Up to a rescaling of the space variable $x$, we can assume that $\lambda=1$ and that all the eigenvalues of $A(u)$ lie in the interval $(-1,1)$, so that the above stability condition is satisfied.

The proof of well-posedness for the system $(1.2)$ is similar to $[1,6]$ : the main step is to obtain a uniform estimate on the total variation of the solution $(u, v)$, independent of $\varepsilon$.

Performing the rescaling $t \rightarrow t / \varepsilon, x \rightarrow x / \varepsilon$, the relaxation system (1.2) becomes

$$
\left\{\begin{array}{l}
u_{t}+v_{x}=0 \\
v_{t}+u_{x}=F(u)-v
\end{array}\right.
$$

We denote with $-1<\lambda_{1}(u)<\cdots<\lambda_{n}(u)<1$ the eigenvalues of the matrix $A(u)$, and call $l^{1}, \ldots, l^{n}, r_{1}, \ldots, r_{n}$ its eigenvectors normalized so that

$$
\left|r_{i}(u)\right|=1, \quad\left\langle l^{i}(u), r_{j}(u)\right\rangle=\delta_{i j} .
$$


Here $\langle\cdot, \cdot\rangle$ is the duality product in $\mathbb{R}^{n}$ and $\delta_{i j}$ is the Kronecker symbol. The directional derivative of a function $\phi=\phi(u)$ in the direction of the eigenvector $r_{i}$ will be written as

$$
r_{i} \bullet \phi(u) \doteq \lim _{h \rightarrow 0} \frac{\phi\left(u+h r_{i}(u)\right)-\phi(u)}{h} .
$$

By defining $z^{+} \doteq u+v, z^{-} \doteq u-v$, we can rewrite $\left(1.2^{\prime}\right)$ as

$$
\left\{\begin{array}{l}
z_{t}^{-}-z_{x}^{-}=-F(u)-\frac{z^{-}}{2}+\frac{z^{+}}{2} \\
z_{t}^{+}+z_{x}^{+}=F(u)+\frac{z^{-}}{2}-\frac{z^{+}}{2}
\end{array}\right.
$$

We now decompose the gradients $z_{x}^{ \pm}$along the eigenvectors $r_{i}$, setting $z_{x}^{ \pm} \doteq \sum f^{j \pm} r_{j}(u)$. From (1.4) we deduce

$$
\begin{aligned}
z_{x t}^{ \pm} & =\sum_{j} f_{t}^{j \pm} r_{j}+\sum_{j} f^{j \pm} u_{t} \bullet r_{j} \\
& =\sum_{j} f^{j \pm} r_{j}-\sum_{j, k} f^{j \pm}\left(\frac{f^{k+}-f^{k-}}{2}\right) r_{k} \bullet r_{j}, \\
z_{x x}^{ \pm} & =\sum_{j} f_{x}^{j \pm} r_{j}+\sum_{j, k} f^{j \pm}\left(\frac{f^{k+}+f^{k-}}{2}\right) r_{k} \bullet r_{j},
\end{aligned}
$$

so that the equations satisfied by the components $f^{i \pm}$ are

$$
\left\{\begin{array}{l}
f_{t}^{i-}-f_{x}^{i-}=-\frac{1+\lambda_{i}(u)}{2} f^{i-}+\frac{1-\lambda_{i}(u)}{2} f^{i+}+\sum_{j, k}\left\langle l^{i}, r_{k} \bullet r_{j}\right\rangle f^{j-} f^{k+}, \\
f_{t}^{i+}+f_{x}^{i+}=+\frac{1+\lambda_{i}(u)}{2} f^{i-}-\frac{1-\lambda_{i}(u)}{2} f^{i+}-\sum_{j, k}\left\langle l^{i}, r_{k} \bullet r_{j}\right\rangle f^{j+} f^{k-} .
\end{array}\right.
$$

Observe that (1.5) consists of $n$ systems of $2 \times 2$ balance laws, coupled through the terms $\left\langle l^{i}, r_{k} \bullet r_{j}\right\rangle f^{j \pm} f^{k \mp}$. As in [1], these terms can be classified as:

- transversal terms: $\left\langle l^{i}, r_{k} \bullet r_{j}\right\rangle f^{j \pm} f^{k \mp}, j, k=1, \ldots, n, j \neq k$;

- non transversal terms: $\left\langle l^{i}, r_{j} \bullet r_{j}\right\rangle f^{j \pm} f^{j \mp}, j=1, \ldots, n$.

In this paper we assume that $r_{i} \bullet r_{i}=0$ for all $i=1, \ldots, n$, i.e. the integral curves of the eigenvector $r_{k}$ are straight lines: this implies that in (1.5) only transversal terms are present. Since the system (1.4) is semilinear, solutions initially smooth remain smooth for all times. Uniform BV bounds on $z^{ \pm}$can thus be obtained from $L^{1}$ estimates on the gradient components $f^{i \pm}$. As in [1,6], towards these estimates the main task is to prove that the $L^{1}$ norm of the coupling terms over the half plane $(t, x) \in \mathbb{R}^{+} \times \mathbb{R}$ is bounded and of quadratic order.

The main novelty of this paper is the introduction of a Glimm-type interaction potential $\mathcal{Q}\left(z^{-}, z^{+}\right)$for the system (1.4). As in [6], one can interpret a solution of the $2 \times 2$ system $(1.5)$ as the density of random particles, whose average speed is $\lambda_{i}(u)$. The potential $\mathcal{Q}$ then represents the expected number of future crossings between particles 
with different speeds. Following this interpretation, $\mathcal{Q}$ provides us the counterpart of the Glimm interaction potential measuring the sum of all approaching waves of different characteristic families [3]. Differently form the hyperbolic case, however, in our system each wave is approaching the others, because of the diffusive behavior of $\left(1.2^{\prime}\right)$, but its strength is weighted by an exponentially decreasing function, measuring the probability of interaction when the particles start at different places.

Our main results are as follows. We first consider the Cauchy problem for the system $\left(1.2^{\prime}\right)$. We assume that the Jacobian matrix $A(u)=D F(u)$, is a smooth uniformly strictly hyperbolic function with values in $\mathbb{R}^{n} \times \mathbb{R}^{n}$, i.e. it has $n$ real distinct eigenvalues $\lambda_{i}$ such that $\lambda_{1}\left(u_{1}\right)<\cdots<\lambda_{n}\left(u_{n}\right)$ for all $u_{1}, \ldots, u_{n} \in \Omega$. By $l^{i}, r_{i}$ we denote its left and right eigenvectors normalized as in (1.3). For a BV function $w: \mathbb{R} \mapsto \Omega$ we denote with Tot.Var. $(w)$ the sum of the total variation of its components along the eigenvectors $r_{i}$,

$$
\text { Tot.Var. }(w) \doteq \sum_{i} \text { Tot.Var. }\left(\left\langle l^{i}, w_{x}\right\rangle\right)
$$

and we write $w(-\infty) \doteq \lim _{x \rightarrow-\infty} w(x)$. Given two BV functions $w_{1}, w_{2}: \mathbb{R} \rightarrow \Omega$, we define the functional $\mathcal{V}\left(w_{1}, w_{2}\right)$ as

$$
\mathcal{V}\left(w_{1}, w_{2}\right) \doteq \operatorname{Tot} . \operatorname{Var} .\left(w_{1}\right)+\operatorname{Tot} . \operatorname{Var} .\left(w_{2}\right)
$$

Our first theorem is concerned with the existence of a global BV solution for (1.4).

THEOREM 1.- For every compact set $K \subset \Omega$ and constant $M>0$ there exist constants $\delta_{0}, C_{0}$, a closed domain $\mathcal{D} \subseteq L_{\text {loc }}^{1}\left(\mathbb{R} ; \mathbb{R}^{2 n}\right)$ and a continuous semigroup $\mathcal{S}: \mathcal{D} \times[0,+\infty) \rightarrow \mathcal{D}$ with the following properties:

(i) the domain $\mathcal{D}$ has the form

$$
\begin{aligned}
& \mathcal{D}=\operatorname{cl}\left\{\left(z^{-}, z^{+}\right) \in W_{\mathrm{loc}}^{1,1}\left(\mathbb{R} ; \mathbb{R}^{2 n}\right):\left(z^{-}+z^{+}\right)(-\infty) \in K,\right. \\
&\left.\left|z^{-}(-\infty)\right|,\left|z^{+}(-\infty)\right| \leqslant M, \mathcal{V}\left(z^{-}, z^{+}\right)+C_{0} \mathcal{Q}\left(z^{-}, z^{+}\right) \leqslant \delta_{0}\right\},
\end{aligned}
$$

where the closure is taken with respect to the $L^{1}$ norm;

(ii) for every initial data

$$
\left(z^{-}(0, x), z^{+}(0, x)\right) \doteq\left(z_{0}^{-}(x), z_{0}^{+}(x)\right) \in \mathcal{D},
$$

there exists a unique globally defined solution to (1.4)-(1.9), corresponding to the semigroup trajectory $t \rightarrow\left(z^{-}(t), z^{+}(t)\right)=\mathcal{S}_{t}\left(z_{0}^{-}, z_{0}^{+}\right)$;

(iii) there exist constants $L, L^{\prime}$ such that for every pair of initial data $\left(z_{0}^{-}, z_{0}^{+}\right)$, $\left(\tilde{z}_{0}^{-}, \tilde{z}_{0}^{+}\right) \in \mathcal{D}$, and for every $s, t \geqslant 0$ one has

$$
\left\|\mathcal{S}_{t}\left(z_{0}^{-}, z_{0}^{+}\right)-\mathcal{S}_{s}\left(\tilde{z}_{0}^{-}, \tilde{z}_{0}^{+}\right)\right\|_{L^{1}} \leqslant L^{\prime}|t-s|+L\left\|\left(z_{0}^{-}, z_{0}^{+}\right)-\left(\tilde{z}_{0}^{-}, \tilde{z}_{0}^{+}\right)\right\|_{L^{1}}
$$

We remark that the domain (1.8) depends only on the total variation of the initial data $\left(z_{0}^{-}, z_{0}^{+}\right)$, and is invariant with respect to to the hyperbolic rescaling $t \rightarrow \varepsilon t, x \rightarrow \varepsilon x$. The above result thus yields an a priori bound on the total variation of solutions $\left(u^{\varepsilon}(t), v^{\varepsilon}(t)\right)$ of (1.2), independent of the parameter $\varepsilon$. 
Our second main result shows that, as $\varepsilon \rightarrow 0+$, these solutions $\left(u^{\varepsilon}(t), v^{\varepsilon}(t)\right)$ converge to a unique limit $(u(t), v(t))$, depending continuously on the initial data $u(0)$.

THEOREM 2. - In the same setting of Theorem 1, there exist constants $L, L^{\prime}, \delta^{\prime}>0$, a closed domain $\mathcal{D}^{\prime} \subset L_{\text {loc }}^{1}$ and a continuous semigroup $S: \mathcal{D}^{\prime} \times\left[0, \infty\left[\mapsto \mathcal{D}^{\prime}\right.\right.$ with the following properties:

(i) the domain $\mathcal{D}^{\prime}$ has the form

$$
\begin{aligned}
\mathcal{D}^{\prime}=\operatorname{cl}\left\{u \in L_{\mathrm{loc}}^{1}\left(\mathbb{R} ; \mathbb{R}^{n}\right):\right. & u(-\infty) \in K, u \text { piecewise constant }, \\
& \left.V(u)+C^{\prime} Q(u) \leqslant \delta^{\prime}\right\},
\end{aligned}
$$

for some constant $C^{\prime}$, where $V(u)$ and $Q(u)$ are the total amount of waves and the Glimm interaction potential measuring the sum of approaching waves of different characteristic families [3];

(ii) for every $\bar{u}, \bar{w} \in \mathcal{D}^{\prime}$ with $\bar{u}-\bar{w} \in L^{1}$ and every $t, s \geqslant 0$ one has

$$
\left\|S_{t} \bar{u}-S_{s} \bar{w}\right\|_{L^{1}} \leqslant L^{\prime}|t-s|+L\|\bar{u}-\bar{w}\|_{L^{1}}
$$

(iii) for every piecewise constant initial data $\bar{u} \in \mathcal{D}^{\prime}$, there exists $\tau>0$ such that the following holds. For every $\bar{u} \in \mathcal{D}^{\prime}$, the trajectory $t \rightarrow u(t, \cdot)=S_{t} \bar{u}$ is the unique entropic solution of the Cauchy problem (1.1) in the sense of [4];

(iv) for every $\bar{u} \in \mathcal{D}$, the trajectory $u(t, \cdot)=S_{t} \bar{u}$ is the unique limit in $L_{\text {loc }}^{1}$ of the corresponding solutions $u^{\varepsilon}(t, \cdot)$ of the relaxation Cauchy problem (1.2), as $\varepsilon \rightarrow 0+$, with initial data

$$
u(0, x)=\bar{u}(x) \in \mathcal{D}^{\prime}, \quad\left(z^{-}(0, x), z^{+}(0, x)\right) \in \mathcal{D}
$$

Moreover, for every $t>0, v(t, x)=F(u(t, x))$ for a.e. $x \in \mathbb{R}$.

The proofs of the two above theorems are worked out in Sections 2-4. We shall first assume that the initial data are sufficiently regular. The general case then follows by approximation.

In Section 2 we start by considering two linear $2 \times 2$ relaxation system. After proving some easy lemmas, we compute the integral of the tensor product of the two solutions. Two components of this integral have a simple probabilistic interpretation: they measure the expected number of times where one random particle overtakes the other. This computation, differently from $[1,6]$, is carried out using complex analysis and the calculus of residues. Finally we give a simple probabilistic interpretation of the results.

In Section 3, we introduce the Glimm-type potential. We show that this potential controls the growth in total variation of the solution of (1.4), (1.9). This proves the first two statements in Theorem 1. Next, we study the linearized equations (1.4) along a solution $\left(z^{-}(t), z^{+}(t)\right)$. As in [1,6], we show that these equations are of the same kind of (1.5), and thus we can again evaluate the increment of the $L^{1}$ norm of the perturbation using the potential. This concludes the proof of Theorem 1 .

In Section 4 we address the question of convergence of the solution as $\varepsilon \rightarrow 0$, proving Theorem 2. The main arguments are as in [6]. 


\section{Estimate of transversal terms in the linear case}

Aim of this section is to prove some easy properties of the solution of a $2 \times 2$ system of balance laws. These remarks are essentially the same as in [6]. Then we consider two solutions of two distinct $2 \times 2$ linear systems, with strictly different average speeds, and we explicit compute the integral of their product over the half plane $\mathbb{R}^{+} \times \mathbb{R}$. The computation is performed using the calculus of residues [2].

Consider a $2 \times 2$ system of balance laws of the form

$$
\left\{\begin{array}{l}
f_{t}^{-}-f_{x}^{-}=-\alpha(t, x) f^{-}+\beta(t, x) f^{+} \\
f_{t}^{+}+f_{x}^{+}=\alpha(t, x) f^{-}-\beta(t, x) f^{+}
\end{array}\right.
$$

with $0<\alpha(t, x), \beta(t, x)<1, \alpha(t, x)+\beta(t, x)=1$. This system can be interpreted as the motion of a random particle with speed \pm 1 . If the initial datum $\left(f_{0}^{-}, f_{0}^{+}\right)$is positive, it remains positive for all $t \geqslant 0$. Moreover it is easy to show that

$$
\left\{\begin{array}{l}
\left|f^{-}\right|_{t}-\left|f^{-}\right|_{x} \leqslant-\alpha(t, x)\left|f^{-}\right|+\beta(t, x)\left|f^{+}\right|, \\
\left|f^{+}\right|_{t}+\left|f^{+}\right|_{x} \leqslant \alpha(t, x)\left|f^{-}\right|-\beta(t, x)\left|f^{+}\right|
\end{array}\right.
$$

and therefore

$$
\frac{\mathrm{d}}{\mathrm{d} t} \int_{\mathbb{R}}\left(\left|f^{-}(t, x)\right|+\left|f^{+}(t, x)\right|\right) \mathrm{d} x \leqslant 0 .
$$

The above relation implies that the $L^{1}$ norm of the solution is not increasing in time, and it will be useful in the next sections.

A particular but fundamental case is when the functions $\alpha, \beta$ are constant: in this case (2.1) becomes

$$
\left\{\begin{array}{l}
f_{t}^{-}-f_{x}^{-}=-\frac{1+a}{2} f^{-}+\frac{1-a}{2} f^{+}, \\
f_{t}^{+}+f_{x}^{+}=\frac{1+a}{2} f^{-}-\frac{1-a}{2} f^{+},
\end{array}\right.
$$

where $a \doteq \alpha-\beta, \alpha \in(-1,1)$. With the above probabilistic interpretation, we can say that $a$ is the time average on $[0,+\infty)$ of the speed of a particle whose density at the point $(t, x)$ is given by $f^{-}(t, x)+f^{+}(t, x)$ (see [11]).

It is well known that the solution of $\left(2.1^{\prime}\right)$ can be explicitly computed using the Fourier transform: if we write the vector $\left(f^{-}, f^{+}\right)$for any fixed $t \geqslant 0$ as

$$
\left[\begin{array}{l}
f^{-}(t, x) \\
f^{+}(t, x)
\end{array}\right] \doteq \frac{1}{\sqrt{2 \pi}} \int_{\mathbb{R}} \mathbf{c}_{\xi}(t) \exp \{-i \xi x\} \mathrm{d} \xi, \quad \mathbf{c}_{\xi}(t) \in \mathbb{R}^{2},
$$


the substitution of the above formula in $\left(2.1^{\prime}\right)$ gives the equations for the Fourier components $\mathbf{c}(t)$ :

$$
\dot{\mathbf{c}}_{\xi}=\left[\begin{array}{cc}
-\frac{1+a}{2}-i \xi & \frac{1-a}{2} \\
\frac{1+a}{2} & -\frac{1-a}{2}+i \xi
\end{array}\right] \mathbf{c}_{\xi} \doteq A(a ; \xi) \mathbf{c}_{\xi} .
$$

We shall denote with

$$
\begin{aligned}
& \sigma_{i}(a ; \xi), \quad r_{i}(a ; \xi)=\left[\begin{array}{c}
r_{i-}(a ; \xi) \\
r_{i+}(a ; \xi)
\end{array}\right], \\
& l^{i}(a ; \xi)=\left[l_{-}^{i}(a ; \xi), l_{+}^{i}(a ; \xi)\right], \quad i=1,2,
\end{aligned}
$$

respectively the $i$ th-eigenvalue and the $i$ th right and left eigenvectors of the matrix $A(u)$, normalized so that

$$
r_{i-}+r_{i+}=1, \quad\left\langle l^{i}(a ; \xi), r_{i}(a ; \xi)\right\rangle \doteq l_{-}^{i} r_{i-}+l_{+}^{i} r_{i+}=1 .
$$

It is easy to prove that, for $\xi \in \mathbb{R} \backslash\{0\}$, one has $-1<\operatorname{Re}(\sigma)<0$ and $\sigma_{1}(a ; 0)=-1$, $\sigma_{2}(a, 0)=0$. The non-positivity of these eigenvalues reflects the fact that the $L^{1}$ norm of a solution is non-increasing.

Finally, if $\hat{f}_{0}(\xi)$ is the Fourier transform of the initial datum $f(0, x)$, the solution to $\left(2.1^{\prime}\right)$ can be written as

$$
\begin{aligned}
{\left[\begin{array}{l}
f^{-}(t, x) \\
f^{+}(t, x)
\end{array}\right]=} & \frac{1}{\sqrt{2 \pi}} \int_{\mathbb{R}}\left\{\left\langle l^{1}(a ; \xi), \hat{f}_{0}(\xi)\right\rangle r_{1}(a ; \xi) \exp \left\{-i \xi x+\sigma_{1}(a ; \xi) t\right\}\right. \\
& \left.+\left\langle l^{2}(a ; \xi), \hat{f}_{0}(\xi)\right\rangle r_{2}(a ; \xi) \exp \left\{-i \xi x+\sigma_{2}(a ; \xi) t\right\}\right\} \mathrm{d} \xi .
\end{aligned}
$$

It will be convenient to study $\sigma$ as a multivalued function on the whole complex plane, defined by the equation

$$
\operatorname{det}[\sigma I-A(a, \xi)]=\sigma^{2}+\sigma-i \xi a+\xi^{2}=0,
$$

In this case the branch points are

$$
\xi_{ \pm}(a)= \pm \frac{\sqrt{1-a^{2}}}{2}+i \frac{a}{2}
$$

and the picture in the complex plane is given in Fig. 1, with $a>0$.

The eigenvalues can be written as

$$
\begin{aligned}
& \sigma_{1}(a ; \xi)=-\frac{1}{2}+i \sqrt{\left|\xi-\xi_{-}\right|\left|\xi-\xi_{+}\right|} \exp \left\{\frac{\theta_{-}+\theta_{+}}{2}\right\}, \\
& \sigma_{2}(a ; \xi)=-\frac{1}{2}-i \sqrt{\left|\xi-\xi_{-}\right|\left|\xi-\xi_{+}\right|} \exp \left\{\frac{\theta_{-}+\theta_{+}}{2}\right\},
\end{aligned}
$$



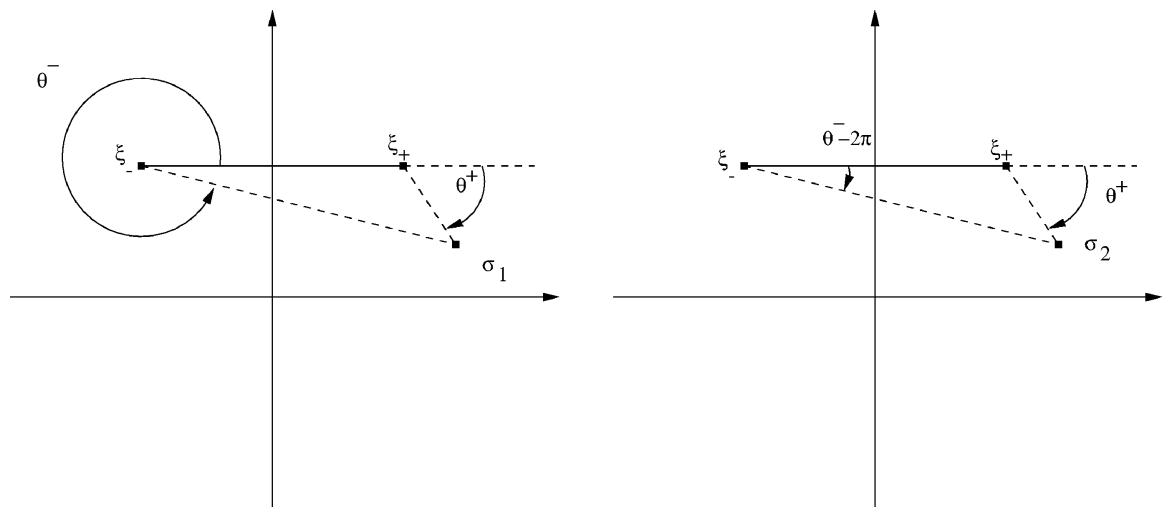

Fig. 1. The two branches of the solution of (2.7), given by (2.8).

where now the square root is considered as a function from $\mathbb{R}^{+}$to $\mathbb{R}^{+}$. Note that, by (2.5), the eigenvectors are rational functions of the eigenvalues, and therefore they have the same branch points.

Consider now two systems of the form $\left(2.1^{\prime}\right)$ with $a^{\prime} \neq a$. We want to compute the (tensor) transversal integral

$$
\int_{0}^{+\infty} \int_{\mathbb{R}}\left[\begin{array}{l}
f^{-}(t, x) \\
f^{+}(t, x)
\end{array}\right] \otimes\left[\begin{array}{l}
\left(f^{-}\right)^{\prime}(t, x) \\
\left(f^{+}\right)^{\prime}(t, x)
\end{array}\right] \mathrm{d} x \mathrm{~d} t,
$$

where we denote with $\left(f^{\mp}\right)^{\prime}(t, x) \in \mathbb{R}^{2}$ the solution of a second system, with $a$ replaced by some different value $a^{\prime}$. The tensor product in (2.9) is defined as

$$
\left[\begin{array}{l}
f^{-} \\
f^{+}
\end{array}\right] \otimes\left[\begin{array}{l}
\left(f^{-}\right)^{\prime} \\
\left(f^{+}\right)^{\prime}
\end{array}\right] \doteq\left[\begin{array}{ll}
f^{-}\left(f^{-}\right)^{\prime} & f^{-}\left(f^{+}\right)^{\prime} \\
f^{+}\left(f^{-}\right)^{\prime} & f^{+}\left(f^{+}\right)^{\prime}
\end{array}\right] .
$$

For the sake of definiteness in the following we assume $a>a^{\prime} \geqslant 0$, the other cases can be handled similarly, and we will denote all quantities referring to this second system by a prime.

If we assume that the initial data $f(0, x)=f_{0}(x), f^{\prime}(0, x)=f_{0}^{\prime}(x)$ are in $L^{2}\left(\mathbb{R} ; \mathbb{R}^{2}\right)$, we can rewrite the integral (2.9) using the Fourier transform as the limit $T \rightarrow+\infty$ of

$$
\begin{aligned}
& \int_{0}^{T} \int_{\mathbb{R}}\left[\begin{array}{l}
f^{-}(t, x) \\
f^{+}(t, x)
\end{array}\right] \otimes\left[\begin{array}{l}
\left(f^{-}\right)^{\prime}(t, x) \\
\left(f^{+}\right)^{\prime}(t, x)
\end{array}\right] \mathrm{d} x \mathrm{~d} t \\
& \quad=\int_{\mathbb{R}} \mathrm{d} \xi \sum_{i, j=1}^{2} \mathbf{c}_{i}(a ; \xi) \otimes \mathbf{c}_{j}^{\prime}\left(a^{\prime} ;-\xi\right) \int_{0}^{T} \mathrm{~d} t \exp \left\{\left(\sigma_{i}(a ; \xi)+\sigma_{j}^{\prime}\left(a^{\prime} ;-\xi\right)\right) t\right\} \\
& \quad=\int_{\mathbb{R}} \mathrm{d} \xi \sum_{i, j=1}^{2} \mathbf{c}_{i}(a ; \xi) \otimes \mathbf{c}_{j}^{\prime}\left(a^{\prime} ;-\xi\right) \frac{\exp \left\{\left(\sigma_{i}(a ; \xi)+\sigma_{j}^{\prime}\left(a^{\prime} ;-\xi\right)\right) T\right\}-1}{\sigma_{i}(a ; \xi)+\sigma_{j}^{\prime}\left(a^{\prime} ;-\xi\right)},
\end{aligned}
$$

where the coefficients $\mathbf{c}_{i}(a ; \xi), \mathbf{c}_{j}^{\prime}\left(a^{\prime} ; \xi\right)$ are in $L^{2}\left(\mathbb{R} ; \mathbb{C}^{2}\right)$. 


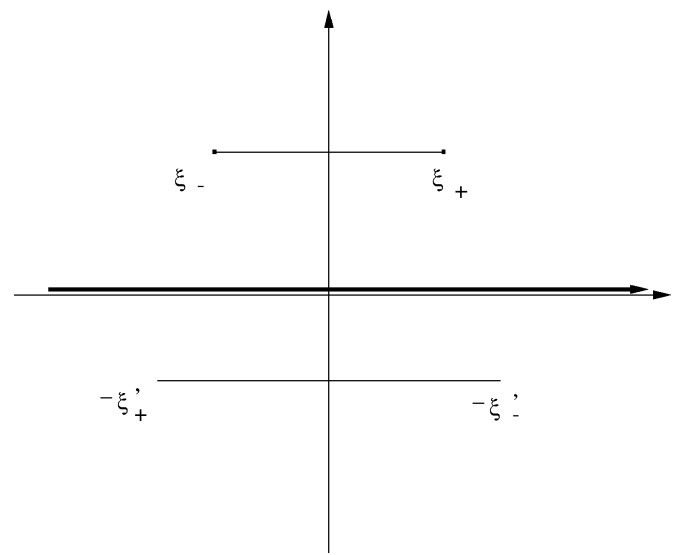

Fig. 2.

Instead of computing the above integral for any initial data $f_{0}, f_{0}^{\prime}$, by the linearity of the systems we only need to compute (2.10) for $f_{0}(x)=\mathbf{v} \delta(x), f_{0}^{\prime}(x)=\mathbf{v}^{\prime} \delta\left(x-x_{0}\right)$, with $\mathbf{v}=\left[v_{1}, v_{2}\right] \in \mathbb{R}^{2}, \mathbf{v}^{\prime}=\left[v_{1}^{\prime}, v_{2}^{\prime}\right] \in \mathbb{R}^{2}$. In this case, by the above analysis and (2.5), the Fourier coefficients $\mathbf{c}, \mathbf{c}^{\prime}$ are multivalued holomorphic functions on the complex plane. However one should consider the principal value of the integral (2.10), i.e. we suppose to move the integral path of $\pm i \varepsilon$ at $\infty$, and then we let $\varepsilon$ go to zero. The choice of the sign will depend on the sign of $x_{0}$ : this assures the exponential decay at $\infty$, i.e. the existence of the integral (2.10). The path of integration in the complex plane is represented in Fig. 2.

To pass to the limit in (2.10), we consider the region $\Omega \subseteq \mathbb{C}$ in which $\operatorname{Re}\left(\sigma_{i}(a ; \xi)+\right.$ $\left.\sigma_{j}^{\prime}\left(a^{\prime} ;-\xi\right)\right)<0, \forall i, j=1,2$ : it can be shown that this region is shaped as in Fig. 3, where

$$
\bar{\xi} \doteq \frac{i}{2} \frac{a-a^{\prime}}{1-\left(\frac{a+a^{\prime}}{2}\right)^{2}}>0 .
$$

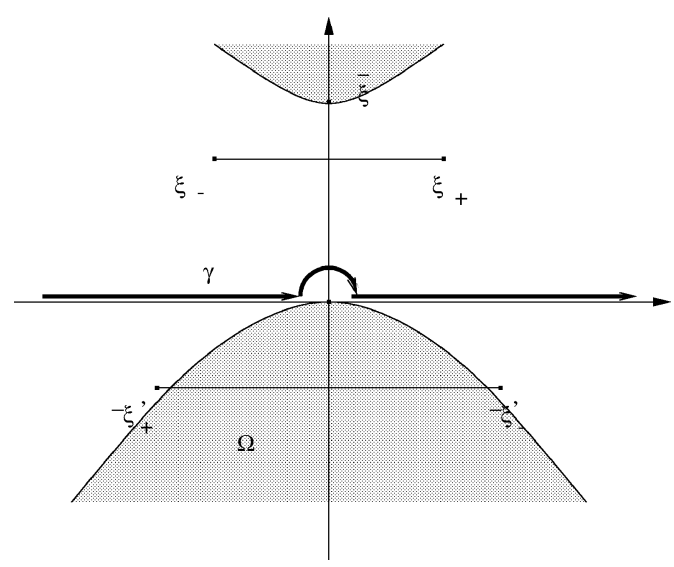

Fig. 3. 
Since $0 \in \Omega$, in order to compute the limit of (2.10) as $T \rightarrow+\infty$, we need to change the path of integration. Noting that the Fourier coefficients of $f_{0}^{\prime}$ can be written as $\mathbf{c}^{\prime}\left(a^{\prime} ; \xi\right) \mathrm{e}^{i \xi x_{0}}$, it follows

$$
\begin{aligned}
& \lim _{T \rightarrow+\infty} \int_{0}^{T} \int_{\mathbb{R}}\left[\begin{array}{l}
f^{-}(t, x) \\
f^{+}(t, x)
\end{array}\right] \otimes\left[\begin{array}{c}
\left(f^{-}\right)^{\prime}(t, x) \\
\left(f^{+}\right)^{\prime}(t, x)
\end{array}\right] \mathrm{d} x \mathrm{~d} t \\
& =\text { p.v. } \int_{\gamma} \sum_{i, j=1}^{2} \frac{\mathbf{c}_{i}(a ; \xi) \otimes \mathbf{c}_{j}^{\prime}\left(a^{\prime} ;-\xi\right)}{\sigma_{i}(a ; \xi)+\sigma_{j}^{\prime}\left(a^{\prime} ;-\xi\right)} \mathrm{e}^{-i \xi x_{0}} \mathrm{~d} \xi \\
& =\text { p.v. } \int_{\widetilde{\gamma}} \frac{\mathbf{c}(a ; \xi) \otimes \mathbf{c}^{\prime}\left(a^{\prime} ;-\xi\right)}{\sigma(a ; \xi)+\sigma^{\prime}\left(a^{\prime} ;-\xi\right)} \mathrm{e}^{-i \xi x_{0}} \mathrm{~d} \xi \doteq \text { p.v. } \int_{\hat{\gamma}} h\left(x_{0} ; \xi\right) \mathrm{d} \xi .
\end{aligned}
$$

Here $\gamma$ is the path in the complex plane depicted in Fig. 3, while $\hat{\gamma}$ is the path $\gamma$ repeated on the Riemann surface made of 4 copies of the complex plane connected by the two cuts, as in Fig. 4.

At this point we need to consider 2 cases separately, corresponding to the sign of $x_{0}$.

Case 1. We suppose $x_{0}<0$, i.e. the particle with average speed $a$ is on the right of the particle with average speed $a^{\prime}$. In this case the integral (2.12) is computed as

$$
\text { p.v. } \int_{\hat{\gamma}} h\left(x_{0} ; \xi\right) \mathrm{d} \xi \doteq \lim _{\varepsilon \rightarrow 0} \int_{\hat{\gamma}+i \varepsilon} h\left(x_{0} ; \xi\right) \mathrm{d} \xi=\operatorname{Res}(h ; \bar{\xi})+\sum_{i=1}^{4} \int_{\gamma_{i}} h\left(x_{0} ; \xi\right) \mathrm{d} \xi,
$$

where $\gamma_{i}, i=1, \ldots, 4$, is one of the 4 paths winding twice a branch point (Fig. 5).

Note that in the neighborhood of any point $\xi_{ \pm}$the variable $(\sigma+1 / 2)^{2}$ can be used to perform the integral along the corresponding $\gamma_{i}$. With this substitution we have

$$
\int_{\gamma_{i}} h\left(x_{0} ; \xi\right) \mathrm{d} \xi=\int_{\eta} h\left(x_{0} ; \xi\right) \xi^{\prime}\left((\sigma+1 / 2)^{2}\right) 2(\sigma+1 / 2) \mathrm{d}(\sigma+1 / 2),
$$

where $\eta$ is a circumference centered at the origin with sufficiently small radius. A simple analysis now shows that the function $h\left(x_{0} ; \xi\right)(\sigma+1 / 2)$ is bounded in the neighborhood of the corresponding branch point, so that the above integrals are equal to 0 for all $i=1, \ldots, 4$. Therefore by calculus of residues (2.13) becomes

$$
\begin{aligned}
& \text { p.v. } \int_{\hat{\gamma}} h\left(x_{0} ; \xi\right) \mathrm{d} \xi=\operatorname{Res}(h ; \bar{\xi}) \\
& =\frac{1}{a-a^{\prime}}\left(\frac{1+a}{2+a+a^{\prime}} v_{1}+\frac{1-a}{2-a-a^{\prime}} v_{2}\right)\left(\frac{1+a^{\prime}}{2+a+a^{\prime}} v_{1}^{\prime}+\frac{1-a^{\prime}}{2-a-a^{\prime}} v_{2}^{\prime}\right) \\
& \quad \times \frac{1}{4}\left[\begin{array}{ll}
\left(2-a-a^{\prime}\right)^{2} & 4-\left(a+a^{\prime}\right)^{2} \\
4-\left(a+a^{\prime}\right)^{2} & \left(2+a+a^{\prime}\right)^{2}
\end{array}\right] \exp \left\{-\frac{2\left(a-a^{\prime}\right)}{4-\left(a+a^{\prime}\right)^{2}} x_{0}\right\} .
\end{aligned}
$$

Case 2. In this case $x_{0}>0$, so that the path of integration $\hat{\gamma}$ is moved in the lower half of the complex plane for large $\xi$. A computation similar to the one above gives 

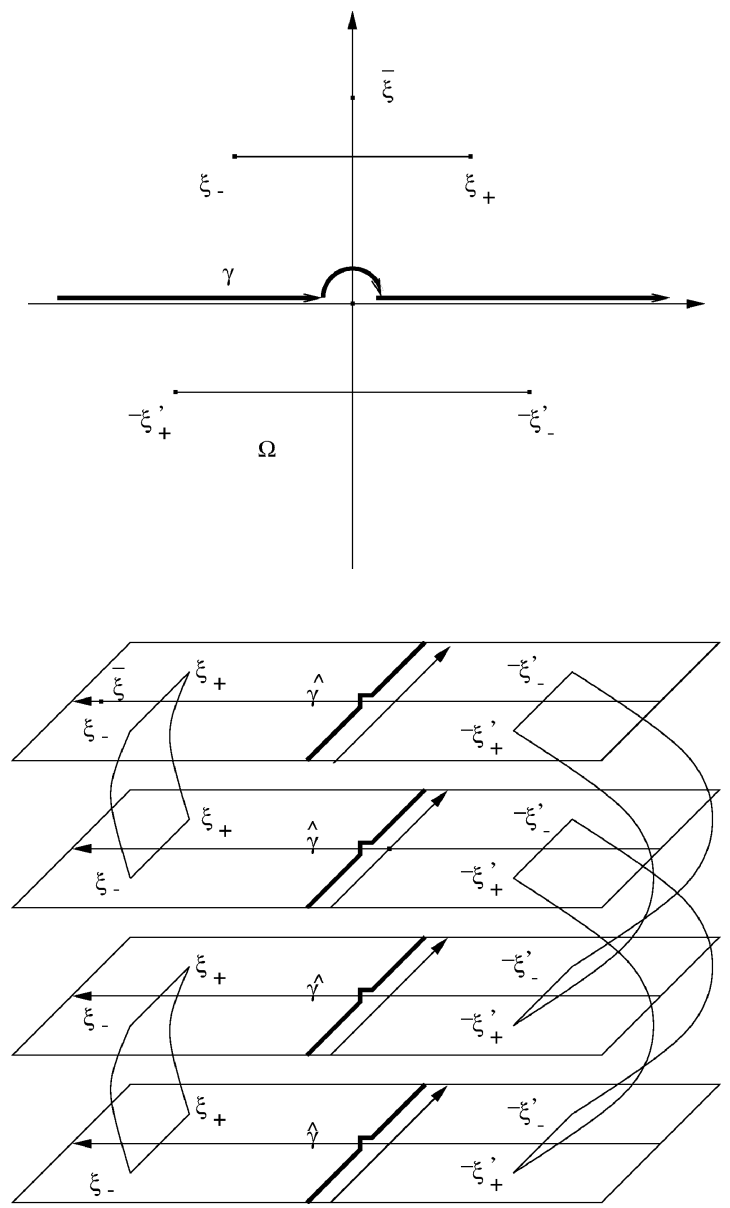

Fig. 4. The path of integration projected on the complex plane and on the Riemann surface.

$$
\begin{aligned}
& \text { p.v. } \int_{\hat{\gamma}} h\left(x_{0} ; \xi\right) \mathrm{d} \xi=\operatorname{Res}(h ; 0) \\
& \quad=\frac{1}{a-a^{\prime}}\left(v_{1}+v_{2}\right)\left(v_{1}^{\prime}+v_{2}^{\prime}\right) \frac{1}{4}\left[\begin{array}{cc}
(1-a)\left(1-a^{\prime}\right) & (1-a)\left(1+a^{\prime}\right) \\
(1+a)\left(1-a^{\prime}\right) & (1+a)\left(1+a^{\prime}\right)
\end{array}\right],
\end{aligned}
$$

Note that in both cases the denominator contains the difference in the average speed of the two particles: this correspond exactly to the computation made in [1].

If now we denote with $P_{i j k l}\left(x_{0}\right)$ the $4 \times 4$ tensor whose action on $\mathbf{v} \otimes \mathbf{v}^{\prime}$ is defined at (2.14) and (2.15), then it is clear that for all $f_{0}, f_{0}^{\prime}>0 \in L^{1}$ we have

$$
\left(\int_{\mathbb{R}^{+}} \int_{\mathbb{R}} f(t, x) \otimes f^{\prime}(t, x) \mathrm{d} x \mathrm{~d} t\right)_{i j}=\int_{\mathbb{R}} \int_{\mathbb{R}} \sum_{k, l=1,2} P_{i j k l}(x-y)\left(f_{0}(x)\right)_{k}\left(f_{0}^{\prime}(y)\right)_{l} \mathrm{~d} x \mathrm{~d} y .
$$

The last part of this section is devoted to some probabilistic computations which explain and simplify the results (2.14)-(2.15). 



Fig. 5.

Following [6], we define

$$
E_{0} \doteq \frac{(1-a)\left(1+a^{\prime}\right)}{4\left(a-a^{\prime}\right)} \quad \text { and } \quad E_{1} \doteq \frac{(1+a)\left(1-a^{\prime}\right)}{4\left(a-a^{\prime}\right)} .
$$

We observe that the quantities $2 E_{0}$ and $2 E_{1}$ yield the expected number of times where the slow particle, starting in front of the fast particle, overtakes or is overtaken by the fast particle, respectively: in fact in each collision the difference in speed is 2 , so that each collision contributes to the integral (2.9) by an amount of $1 / 2$. The relation $2 E_{0}+1=2 E_{1}$ stems from the fact that the fast particle eventually overtakes the slow particle with probability 1 .

We note that, in (2.15), the only coefficient depending of the initial data has the form $\left(v_{1}+v_{2}\right)\left(v_{1}^{\prime}+v_{2}^{\prime}\right)$ and that (2.15) does not depend on $x_{0}$ : this follows from the fact that the fast particle, starting behind the slow particle, must collide with the slow one, no matter which is the position and the initial speed. We can thus rewrite (2.15) as

$$
\operatorname{Res}(h ; 0)=\left(v_{1}+v_{2}\right)\left(v_{1}^{\prime}+v_{2}^{\prime}\right)\left[\begin{array}{cc}
\cdots & E_{0} \\
E_{1} & \cdots
\end{array}\right] .
$$


To interpret (2.14), we first compute the probability distribution of the first time $X$ where a particle, starting with speed -1 at $t=0$ and satisfying $\left(2.1^{\prime}\right)$, changes speed to +1 . The partition function is easily computed to be

$$
P(X \leqslant t) \doteq \begin{cases}0, & t<0 \\ 1-\exp \left\{-\frac{1+a}{2} t\right\}, & t \geqslant 0 .\end{cases}
$$

Now consider two particles, with average speeds $a$ and $a^{\prime}$, both starting with speed -1 at $t=0$. If $X$ and $Y$ denotes the first time in which they change speed, the probability density $\phi(\cdot)$ of $Z \doteq X-Y$ is

$$
\phi(z)= \begin{cases}\frac{1+a}{2+a+a^{\prime}} \frac{1+a^{\prime}}{2} \exp \left\{-\frac{1+a^{\prime}}{2} z\right\}, \quad z \leqslant 0, \\ \frac{1+a^{\prime}}{2+a+a^{\prime}} \frac{1+a}{2} \exp \left\{-\frac{1+a}{2} z\right\}, \quad z \geqslant 0\end{cases}
$$

so that we define

$$
p_{-} \doteq \frac{1+a}{2+a+a^{\prime}}, \quad p_{-}^{\prime} \doteq \frac{1+a^{\prime}}{2+a+a^{\prime}}=1-p_{-},
$$

as the probabilities that the first particle changes speed before the second one or vice versa. In the case where the particles start with speed +1 , with very similar computations one finds

$$
p_{+} \doteq \frac{1-a}{2-a-a^{\prime}}, \quad p_{+}^{\prime} \doteq \frac{1-a^{\prime}}{2-a-a^{\prime}}=1-p_{+} .
$$

Note that $p_{+}<p_{-}, p_{-}^{\prime}<p_{+}^{\prime}$.

Using (2.18) and (2.19) and assuming $x_{0}=0$, we can write the right hand side of (2.14) as

$$
\operatorname{Res}(h ; \bar{x} i)=\left(v_{1}+\frac{p_{+}}{p_{-}} v_{2}\right)\left(\frac{p_{-}^{\prime}}{p_{+}^{\prime}} v_{1}^{\prime}+v_{2}^{\prime}\right)\left[\begin{array}{cc}
\cdots & E_{1} \\
E_{1} & \ldots
\end{array}\right],
$$

where we use the relation

$$
\frac{E_{0}}{E_{1}}=\frac{p_{+} p_{-}^{\prime}}{p_{-} p_{+}^{\prime}} .
$$

We now explain the element of the matrix corresponding to the collisions in which the slow particle overtakes the fast. Using (2.20) we have

$$
\begin{aligned}
& E_{1}\left(v_{1}+\frac{p_{+}}{p_{-}} v_{2}\right)\left(\frac{p_{-}^{\prime}}{p_{+}^{\prime}} v_{1}^{\prime}+v_{2}^{\prime}\right) \\
& =E_{0}\left(\frac{p_{-}}{p_{+}} v_{1}+v_{2}\right)\left(v_{1}^{\prime}+\frac{p_{+}^{\prime}}{p_{-}^{\prime}} v_{2}^{\prime}\right) \\
& =\left[E_{1} p_{-}^{\prime}+E_{0} p_{-}\right] v_{1} v_{1}^{\prime}+E_{1} v_{1} v_{2}^{\prime}+E_{0} v_{2} v_{1}^{\prime}+\left[E_{0} p_{+}^{\prime}+E_{1} p_{+}\right] v_{2} v_{2}^{\prime} .
\end{aligned}
$$



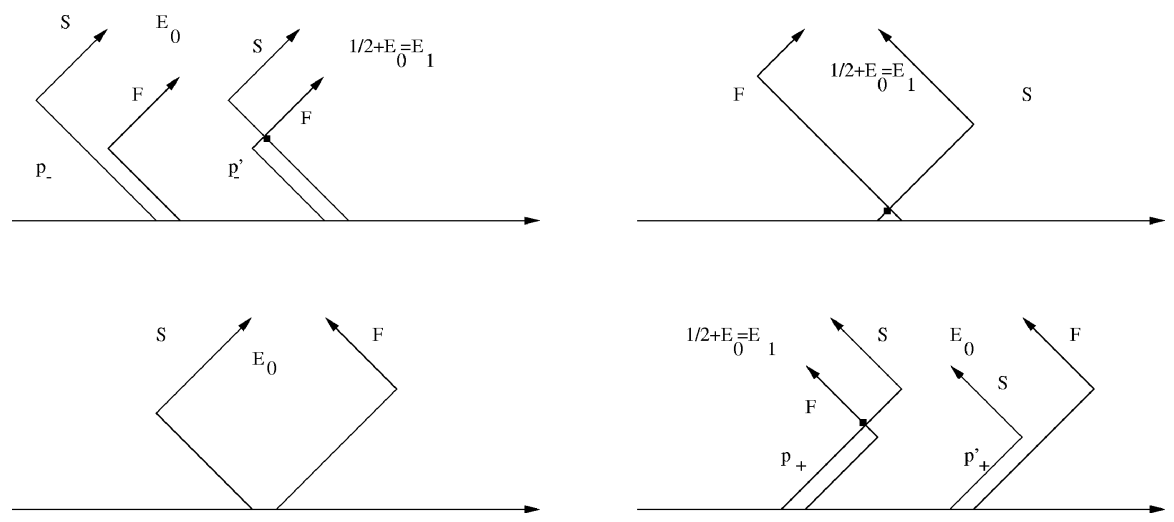

Fig. 6. Possible collisions depending on the initial speeds of the particles.

Each coefficient is the probability that one of the particles changes speed multiplied by the expected number of collisions computed using $\left(2.15^{\prime}\right)$. The various possible cases are illustrated in Fig. 6.

With a very similar analysis, one obtains

$$
\begin{aligned}
& E_{1}\left(v_{1}+\frac{p_{+}}{p_{-}} v_{2}\right)\left(\frac{p_{-}^{\prime}}{p_{+}^{\prime}} v_{1}^{\prime}+v_{2}^{\prime}\right) \\
& \quad=\left[E_{1} p_{-}^{\prime}+E_{0} p_{-}\right] v_{1} v_{1}^{\prime}+E_{1} v_{1} v_{2}^{\prime}+E_{0} v_{2} v_{1}^{\prime}+\left[E_{0} p_{+}^{\prime}+E_{1} p_{+}\right] v_{2} v_{2}^{\prime} .
\end{aligned}
$$

Moreover we have the relations:

$$
\begin{aligned}
& 2 E_{0}=\frac{p_{+} p_{-}^{\prime}}{p_{-} p_{+}^{\prime}-p_{+} p_{-}^{\prime}}, \quad 2 E_{1}=\frac{p_{-} p_{+}^{\prime}}{p_{-} p_{+}^{\prime}-p_{+} p_{-}^{\prime}}, \\
& \alpha \doteq \frac{2\left(a-a^{\prime}\right)}{4-\left(a+a^{\prime}\right)^{2}}=p_{-} p_{+}^{\prime}-p_{+} p_{-}^{\prime},
\end{aligned}
$$

where $\alpha$ is the decay exponent in (2.14).

\section{Proof of Theorem 1}

In this section we prove the first theorem stated in the introduction. The main tool is the introduction of a Glimm type interaction potential which gives a bound on the growth of the total variation of the solution to (1.4). Before proving the result in the general case, we consider the case in which we have two equations of the form $\left(2.1^{\prime}\right)$, with constants $a$ and $a^{\prime}, a \neq a^{\prime}$, and we want to bound the instantaneous interaction,

$$
\int_{\mathbb{R}} f^{-}(t, x)\left(f^{+}\right)^{\prime}(t, x)+f^{+}(t, x)\left(f^{-}\right)^{\prime}(t, x) \mathrm{d} x .
$$

We first recall the basic quantities obtained in the previous section: if $A, A^{\prime}$ are the collision matrices in the right hand side of $\left(2.1^{\prime}\right)$, and $\Lambda$ is the hyperbolic matrix of the principal part, i.e. 
S. BIANCHINI / Ann. Inst. Henri Poincaré Anal. nonlinear 18 (2001) 19-42

$$
\begin{aligned}
& A \doteq\left[\begin{array}{cc}
-\frac{1+a}{2} & \frac{1-a}{2} \\
\frac{1+a}{2} & -\frac{1-a}{2}
\end{array}\right], \quad A^{\prime} \doteq\left[\begin{array}{cc}
-\frac{1+a^{\prime}}{2} & \frac{1-a^{\prime}}{2} \\
\frac{1+a^{\prime}}{2} & -\frac{1-a^{\prime}}{2}
\end{array}\right], \\
& \Lambda \doteq\left[\begin{array}{cc}
-1 & 0 \\
0 & 1
\end{array}\right],
\end{aligned}
$$

then the exponent of decay $\alpha$ and the corresponding eigenvalue $\sigma$ are defined as

$$
\alpha \doteq \frac{2\left(a-a^{\prime}\right)}{4-\left(a+a^{\prime}\right)^{2}}, \quad \sigma \doteq \frac{\left(a-a^{\prime}\right)\left(a+a^{\prime}\right)}{\left(4-\left(a+a^{\prime}\right)^{2}\right)} .
$$

The above definitions implies that the projectors

$$
\begin{array}{ll}
l_{0} \doteq[1,1], & l_{0}^{\prime} \doteq[1,1], \\
l_{1} \doteq\left[p_{-}, p_{+}\right], & l_{1}^{\prime} \doteq\left[p_{-}^{\prime}, p_{+}^{\prime}\right],
\end{array}
$$

satisfy the following relations:

$$
\begin{array}{ll}
l_{0} A=0, & l_{0}^{\prime} A^{\prime}=0, \\
l_{1}(A-\sigma I-\alpha \Lambda)=0, & l_{1}^{\prime}\left(A^{\prime}+\sigma I+\alpha \Lambda\right)=0 .
\end{array}
$$

We recall that the quantities $p_{-}, p_{+}, p_{-}^{\prime}, p_{+}^{\prime}$ are defined in (2.18), (2.19):

$$
\begin{aligned}
& p_{-} \doteq \frac{1+a}{2+a+a^{\prime}}, \quad p_{-}^{\prime} \doteq 1-p_{-} \quad \text { and } \\
& p_{+} \doteq \frac{1-a}{2-a-a^{\prime}}, \quad p_{+}^{\prime} \doteq 1-p_{+} .
\end{aligned}
$$

Remark 3.1. - One can define the quantities $\alpha, \sigma$, and the projectors (3.3) using (3.4). It is clear that the results are exactly the same. However the computation performed in Section 2 can be useful to study the transversal terms in other situation, for example in connection with discrete numerical schemes.

Define the interaction potential $Q\left(f, f^{\prime}\right)$ as

$$
\begin{aligned}
Q\left(f, f^{\prime}\right) \doteq & \iint_{\mathbb{R}^{2}} \sum_{k, l=1}^{2}\left(P_{12 k l}(x-y)+P_{21 k l}(x-y)\right)\left(f_{0}(x)\right)_{i}\left(f_{0}^{\prime}(y)\right)_{j} \mathrm{~d} x \mathrm{~d} y \\
= & \iint_{x<y}\left(E_{0}+E_{1}\right)\left\langle l_{0}, f(t, x)\right\rangle\left\langle l_{0}^{\prime}, f^{\prime}(t, y)\right\rangle \mathrm{d} x \mathrm{~d} y \\
& +\iint_{x>y}\left(\frac{E_{0}}{p_{+} p_{-}^{\prime}}+\frac{E_{1}}{p_{-} p_{+}^{\prime}}\right)\left\langle l_{1}, f(t, x)\right\rangle\left\langle l_{1}^{\prime}, f^{\prime}(t, y)\right\rangle \mathrm{e}^{-\alpha(x-y)} \mathrm{d} x \mathrm{~d} y .
\end{aligned}
$$

For a fixed time $t$, this quantity gives precisely the future expected number of mutual crossings of the two particles. One thus expects that its time derivative coincides with 


$$
-\left\{\int_{\mathbb{R}} f^{-}(t, x)\left(f^{+}\right)^{\prime}(t, x)+f^{+}(t, x)\left(f^{-}\right)^{\prime}(t, x) \mathrm{d} x\right\},
$$

i.e. the instantaneous crossings at time $t$.

Differentiating $Q$ with respect to $t$ one has

$$
\begin{aligned}
\frac{\mathrm{d} Q\left(f, f^{\prime}\right)}{\mathrm{d} t}= & \iint_{x<y}\left(E_{0}+E_{1}\right)\left\langle l_{0}, f_{t}(t, x)\right\rangle\left\langle l_{0}^{\prime}, f(t, y)\right\rangle \mathrm{d} x \mathrm{~d} y \\
& +\iint_{x<y}\left(E_{0}+E_{1}\right)\left\langle l_{0}, f(t, x)\right\rangle\left\langle l_{0}^{\prime}, f_{t}(t, y)\right\rangle \mathrm{d} x \mathrm{~d} y \\
& +\iint_{x>y}\left(\frac{E_{0}}{p_{+} p_{-}^{\prime}}+\frac{E_{1}}{p_{-} p_{+}^{\prime}}\right)\left\langle l_{1}, f_{t}(t, x)\right\rangle\left\langle l_{1}^{\prime}, f^{\prime}(t, y)\right\rangle \mathrm{e}^{-\alpha(x-y)} \mathrm{d} x \mathrm{~d} y \\
& +\iint_{x>y}\left(\frac{E_{0}}{p_{+} p_{-}^{\prime}}+\frac{E_{1}}{p_{-} p_{+}^{\prime}}\right)\left\langle l_{1}, f(t, x)\right\rangle\left\langle l_{1}^{\prime}, f_{t}^{\prime}(t, y)\right\rangle \mathrm{e}^{-\alpha(x-y)} \mathrm{d} x \mathrm{~d} y \\
= & I_{1}+I_{2}+I_{3}+I_{4} .
\end{aligned}
$$

The above integrals will be studied separately. Using $\left(2.1^{\prime}\right)$ and (3.1)-(3.5), one finds

$$
\begin{aligned}
I_{1}= & \left(E_{0}+E_{1}\right) \int_{\mathbb{R}}\left(f^{-}(t, x)-f^{+}(t, x)\right)\left(\left(f^{-}\right)^{\prime}(t, x)+\left(f^{+}\right)^{\prime}(t, x)\right) \mathrm{d} x, \\
I_{2}= & \left(E_{0}+E_{1}\right) \int_{\mathbb{R}}\left(f^{-}(t, x)+f^{+}(t, x)\right)\left(-\left(f^{-}\right)^{\prime}(t, x)+\left(f^{+}\right)^{\prime}(t, x)\right) \mathrm{d} x, \\
I_{3}= & \left(\frac{E_{0}}{p_{+} p_{-}^{\prime}}+\frac{E_{1}}{p_{-} p_{+}^{\prime}}\right) \int_{\mathbb{R}}\left(-p_{-} f^{-}(t, x)+p_{+} f^{+}(t, x)\right) \\
& \times\left(p_{-}^{\prime}\left(f^{-}\right)^{\prime}(t, x)+p_{+}^{\prime}\left(f^{+}\right)^{\prime}(t, x)\right) \mathrm{d} x \\
& +\left(\frac{E_{0}}{p_{+} p_{-}^{\prime}}+\frac{E_{1}}{p_{-} p_{+}^{\prime}}\right) \iint_{x>y} \sigma\left\langle l_{1}, f(t, x)\right\rangle\left\langle l_{1}^{\prime}, f^{\prime}(t, y)\right\rangle \mathrm{e}^{-\alpha(x-y)} \mathrm{d} x \mathrm{~d} y, \\
I_{4}= & \left(\frac{E_{0}}{p_{+} p_{-}^{\prime}}+\frac{E_{1}}{p_{-} p_{+}^{\prime}}\right) \iint_{\mathbb{R}}\left(p_{-} f^{-}(t, x)+p_{+} f^{+}(t, x)\right) \\
& \times\left(p_{-}^{\prime}\left(f^{-}\right)^{\prime}(t, x)-p_{+}^{\prime}\left(f^{+}\right)^{\prime}(t, x)\right) \mathrm{d} x \\
& +\left(\frac{E_{0}}{p_{+} p_{-}^{\prime}}+\frac{E_{1}}{p_{-} p_{+}^{\prime}}\right) \iint_{x>y}-\sigma\left\langle l_{1}, f(t, x)\right\rangle\left\langle l_{1}^{\prime}, f^{\prime}(t, y)\right\rangle \mathrm{e}^{-\alpha(x-y)} \mathrm{d} x \mathrm{~d} y,
\end{aligned}
$$

Summing up all integrals, from (3.7) we obtains

$$
\begin{aligned}
\frac{\mathrm{d} Q(t)}{\mathrm{d} t} & =I_{1}+I_{2}+I_{3}+I_{4} \\
& =\left(E_{0}+E_{1}\right) \int_{\mathbb{R}}\left(2 f^{-}\left(f^{+}\right)^{\prime}-2 f^{+}\left(f^{-}\right)^{\prime}\right) \mathrm{d} x+\left(\frac{E_{0}}{p_{+} p_{-}^{\prime}}+\frac{E_{1}}{p_{-} p_{+}^{\prime}}\right)
\end{aligned}
$$


S. BIANCHINI / Ann. Inst. Henri Poincaré Anal. nonlinear 18 (2001) 19-42

$$
\begin{aligned}
& \times \int_{\mathbb{R}}\left(-2 p_{-} p_{+}^{\prime} f^{-}\left(f^{+}\right)^{\prime}+2 p_{+} p_{-}^{\prime} f^{+}\left(f^{-}\right)^{\prime}\right) \mathrm{d} x \\
= & 2\left(E_{0}+E_{1}-E_{0} \frac{p_{-} p_{+}^{\prime}}{p_{+} p_{-}^{\prime}}-E_{1}\right) f^{-}\left(f^{+}\right)^{\prime} \\
& +2\left(-E_{0}-E_{1}++E_{0}+E_{0} \frac{p_{-}^{\prime} p_{+}}{p_{+}^{\prime} p_{-}}\right) f^{+}\left(f^{-}\right)^{\prime} \\
= & -\int_{\mathbb{R}} f^{-}(t, x)\left(f^{+}\right)^{\prime}(t, x)+f^{+}(t, x)\left(f^{-}\right)^{\prime}(t, x) \mathrm{d} x,
\end{aligned}
$$

because of the relation (2.20). Using again (2.20), we can rewrite the potential (3.6) as

$$
\begin{aligned}
Q\left(f, f^{\prime}\right) \doteq & \iint_{x<y}\left(E_{0}+E_{1}\right)\left(f^{-}+f^{+}\right)\left(\left(f^{-}\right)^{\prime}+\left(f^{+}\right)^{\prime}\right) \mathrm{d} x \mathrm{~d} y \\
& \left.+\iint_{x>y} 2 E_{1}\left(f^{-}+f^{+} \cdot p_{+} / p_{-}\right)\right) \\
& \times\left(\left(f^{-}\right)^{\prime} \cdot p_{-}^{\prime} / p_{+}^{\prime}+\left(f^{+}\right)^{\prime}\right) \mathrm{e}^{-\alpha(x-y)} \mathrm{d} x \mathrm{~d} y,
\end{aligned}
$$

with $p_{+} / p_{-}<1, p_{-}^{\prime} / p_{+}^{\prime}<1$. This proves the estimate

$$
Q\left(f, f^{\prime}\right) \leqslant 2 E_{1} \iint_{\mathbb{R}^{2}}\left(f^{-}+f^{+}\right)\left(\left(f^{-}\right)^{\prime}+\left(f^{+}\right)^{\prime}\right) \mathrm{d} x \mathrm{~d} y .
$$

Formula (3.8) can be better understood by considering two situations (Fig. 7).

(i) The fast particle is overtaking the slow one. In this case the potential before the interaction is $E_{1}$. Afterwards it equals $E_{0}$. Therefore the variation of the potential in a time $\mathrm{d} t$ is given by

$$
\mathrm{d} Q(t)=2\left(E_{1}-E_{0}\right) f^{+}\left(f^{-}\right)^{\prime} \mathrm{d} t=f^{+}\left(f^{-}\right)^{\prime} \mathrm{d} t .
$$

The coefficient 2 is due to the difference in speed of the particles, since in the interval of time $\mathrm{d} t$ they cover a distance $2 \mathrm{~d} t$.

(ii) A slow particle is overtaking the fast one. The computation is exactly the same of (i):

$$
\mathrm{d} Q(t)=2\left(E_{1}-E_{0}\right) f^{-}\left(f^{+}\right)^{\prime} \mathrm{d} t=f^{-}\left(f^{+}\right)^{\prime} \mathrm{d} t .
$$

Now we can handle the general case. We start by choosing constants $c, \delta_{0}$ so that the compact set

$$
K_{1} \doteq\left\{u \in \mathbb{R}^{n} ; \operatorname{dist}(u, K) \leqslant \delta_{0}\right\}
$$

is entirely contained inside $\Omega$, and moreover

$$
\lambda_{j}(u)-\lambda_{i}(v) \geqslant c \quad \text { whenever } i<j, u, v \in K_{1} .
$$



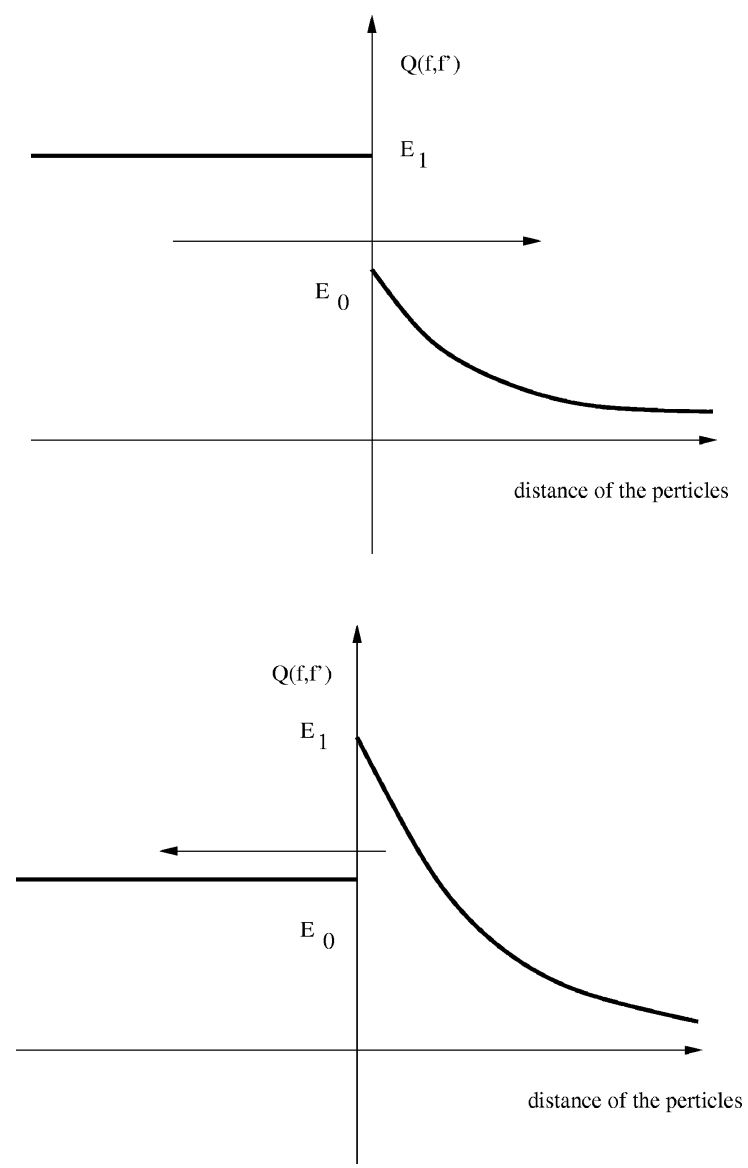

Fig. 7.

We also choose a constant $C_{0}$ such that

$$
\left|\left\langle l^{i}(u), r_{k} \bullet r_{j}(u)\right\rangle\right| \leqslant C_{0} \quad \text { for all } u \in K_{1} \text {. }
$$

Moreover we define

$$
\lambda_{i}^{-}=\min _{u \in K_{1}} \lambda_{i}(u), \quad \lambda_{i}^{+}=\max _{u \in K_{1}} \lambda_{i}(u) \quad \text { for all } i=1, \ldots, n
$$

Using the above definitions and (2.2), from (1.5) it follows that

$$
\left\{\begin{array}{l}
\left|f^{i-}\right|_{t}-\left|f^{i-}\right|_{x} \leqslant-\frac{1+\lambda_{i}(u)}{2}\left|f^{i-}\right|+\frac{1-\lambda_{i}(u)}{2}\left|f^{i+}\right|+C_{0} \sum_{j \neq k}\left|f^{j-}\right|\left|f^{k+}\right|, \\
\left|f^{i+}\right|_{t}+\left|f^{i+}\right|_{x} \leqslant \frac{1+\lambda_{i}(u)}{2}\left|f^{i-}\right|-\frac{1-\lambda_{i}(u)}{2}\left|f^{i+}\right|+C_{0} \sum_{j \neq k}\left|f^{j-}\right|\left|f^{k+}\right| .
\end{array}\right.
$$

We now introduce the interaction potential as 


$$
\begin{aligned}
\mathcal{Q}\left(z^{-}, z^{+}\right) \doteq & \sum_{j>i}\left\{\iint_{x<y}\left(E_{0}^{i j}+E_{1}^{i j}\right)\left\langle l_{0},\left|f^{j}(t, x)\right|\right\rangle\left\langle l_{0},\left|f^{i}(t, y)\right|\right\rangle \mathrm{d} x \mathrm{~d} y\right. \\
& +\iint_{x>y}\left(\frac{E_{0}^{i j}}{p_{+}^{j} p_{-}^{i}}+\frac{E_{1}^{i j}}{p_{-}^{i j}\left(p_{+}^{i j}\right)^{\prime}}\right)\left\langle l_{1}^{i j},\left|f^{j}(t, x)\right|\right\rangle \\
& \left.\times\left\langle\left(l_{1}^{i j}\right)^{\prime},\left|f^{i}(t, y)\right|\right\rangle \mathrm{e}^{-\alpha^{i j}(x-y)} \mathrm{d} x \mathrm{~d} y\right\} \\
= & \sum_{j>i} \mathcal{Q}^{i j}
\end{aligned}
$$

where $f^{i \pm}$ are the components of the spatial derivatives of $z^{-}, z^{+}$defined in the Introduction. For $j>i$, the constants are chosen such that

$$
\begin{gathered}
\alpha^{i j} \doteq \frac{2\left(\lambda_{j}^{-}-\lambda_{i}^{+}\right)}{4-\left(\lambda_{j}^{-}+\lambda_{i}^{+}\right)^{2}}, \quad l_{0} \doteq[1,1], \\
E_{0}^{i j} \doteq \frac{\left(1+\lambda_{i}^{+}\right)\left(1-\lambda_{j}^{-}\right)}{4\left(\lambda_{i}^{+}-\lambda_{j}^{-}\right)}, \quad E_{1}^{i j} \doteq \frac{\left(1-\lambda_{i}^{+}\right)\left(1+\lambda_{j}^{-}\right)}{4\left(\lambda_{i}^{+}-\lambda_{j}^{-}\right)},
\end{gathered}
$$

and the projectors are

$$
\begin{aligned}
l_{1}^{i j} & \doteq\left[\frac{1+\lambda_{j}^{-}}{2+\lambda_{j}^{-}+\lambda_{i}^{+}}, \frac{1-\lambda_{j}^{-}}{2-\lambda_{j}^{-}-\lambda_{i}^{+}}\right] \doteq\left[p_{-}^{i j}, p_{+}^{i j}\right] \\
\left(l_{1}^{i j}\right)^{\prime} & \doteq\left[\frac{1+\lambda_{j}^{-}}{2+\lambda_{j}^{-}+\lambda_{i}^{+}}, \frac{1-\lambda_{j}^{-}}{2-\lambda_{j}^{-}-\lambda_{i}^{+}}\right] \doteq\left[\left(p_{-}^{i j}\right)^{\prime},\left(p_{+}^{i j}\right)^{\prime}\right] .
\end{aligned}
$$

Defining the matrices

$$
\begin{aligned}
& A^{i j} \doteq\left[\begin{array}{cc}
-\frac{1+\lambda_{j}^{-}}{2} & \frac{1-\lambda_{j}^{-}}{2} \\
\frac{1+\lambda_{j}^{-}}{2} & -\frac{1-\lambda_{j}^{-}}{2}
\end{array}\right], \\
& \left(A^{i j}\right)^{\prime} \doteq\left[\begin{array}{cc}
-\frac{1+\lambda_{i}^{+}}{2} & \frac{1-\lambda_{i}^{+}}{2} \\
\frac{1+\lambda_{i}^{+}}{2} & -\frac{1-\lambda_{i}^{+}}{2}
\end{array}\right], \\
& \Lambda \doteq\left[\begin{array}{cc}
-1 & 0 \\
0 & 1
\end{array}\right],
\end{aligned}
$$

we can rewrite (3.12) in vector form for the components $i, j$ as

$$
\begin{aligned}
& \left|f^{i}\right|_{t}+\Lambda\left|f^{i}\right|_{x} \leqslant A^{i j}\left|f^{i}\right|+\left(\lambda_{i}-\lambda_{i}^{+}\right) B\left|f^{i}\right|+s(t, x)[1,1], \\
& \left|f^{j}\right|_{t}+\Lambda\left|f^{j}\right|_{x} \leqslant\left(A^{i j}\right)^{\prime}\left|f^{j}\right|+\left(\lambda_{j}-\lambda_{j}^{-}\right) B\left|f^{j}\right|+s(t, x)[1,1],
\end{aligned}
$$


where

$$
s=C_{0} \sum_{j \neq k}\left|f^{j+}\right| f^{k-} \mid \quad \text { and } \quad B \doteq \frac{1}{2}\left[\begin{array}{cc}
-1 & -1 \\
1 & 1
\end{array}\right],
$$

and $|f|=\left(\left|f^{-}\right|,\left|f^{+}\right|\right)$. The meaning of $\left(3.12^{\prime}\right)$ is that, neglecting the source terms, the particle $i$ will have an average speed lower than $\lambda_{i}^{+}$, while the particle $j$ will travel at least with speed $\lambda_{j}^{-}$. Thus in the coefficients of the potential (3.13) we are assuming the worst case, i.e. for all couple of particles the case in which their speed is closer.

We now compute the derivative of each $Q_{i j}$. With a computation similar to (3.8), we find

$$
\begin{aligned}
\frac{\mathrm{d} \mathcal{Q}_{i j}}{\mathrm{~d} t} \leqslant & -\int_{\mathbb{R}}\left(\left|f^{j-}(t, x)\right|\left|f^{i+}(t, x)\right|+\left|f^{j+}(t, x)\right|\left|f^{i-}(t, x)\right|\right) \mathrm{d} x \\
& +\iint_{x>y}\left(\frac{E_{0}^{i j}}{p_{+}^{i j}\left(p_{-}^{i j}\right)^{\prime}}+\frac{E_{1}^{i j}}{p_{-}^{i j}\left(p_{+}^{i j}\right)^{\prime}}\right)\left(\lambda_{j}-\lambda_{j}^{-}\right)\left(p_{+}^{i j}-p_{-}^{i j}\right)\left|f^{j}(t, x)\right| \\
& \times\left\langle\left(l_{1}^{i j}\right)^{\prime},\left|f^{i}(t, y)\right|\right\rangle \mathrm{e}^{-\alpha^{i j}(x-y)} \mathrm{d} x \mathrm{~d} y \\
& +\iint_{x>y}\left(E_{0}^{i j} p_{+}^{i j}\left(p_{-}^{i j}\right)^{\prime}+\frac{E_{1}^{i j}}{p_{-}^{i j}\left(p_{+}^{i j}\right)^{\prime}}\right)\left\langle l_{1}^{i j},\left|f^{j}(t, y)\right|\right\rangle\left(\lambda_{i}-\lambda_{i}^{+}\right) \\
& \times\left(\left(p_{+}^{i j}\right)^{\prime}-\left(p_{-}^{i j}\right)^{\prime}\right)\left|f^{i}(t, x)\right| \mathrm{e}^{-\alpha^{i j}}(x-y) \mathrm{d} x \mathrm{~d} y \\
& +4 E_{1}^{i j} C_{0} \int_{\mathbb{R}}\left(\left|f^{i}(t, x)\right|+\left|f^{j}(t, x)\right|\right) \mathrm{d} x \int_{\mathbb{R}} s(t, x) \mathrm{d} x \\
\leqslant & \left(\left\|f^{j-}(t) f^{i+}(t)\right\|_{L^{1}}+\left\|f^{j+}(t) f^{i-}(t)\right\|_{L^{1}}\right) \\
& +4 E_{1}^{i j}\left(\left\|f^{i}(t)\right\|_{L^{1}}+\left\|f^{j}(t)\right\|_{L^{1}}\right)\|s(t)\|_{L^{1}},
\end{aligned}
$$

because $\lambda_{j} \geqslant \lambda_{j}^{-}, p_{-}^{i j} \geqslant p_{+}^{i j}$ and $\lambda_{i} \leqslant \lambda_{i}^{+},\left(p_{-}^{i j}\right)^{\prime} \geqslant\left(p_{+}^{i j}\right)^{\prime}$. Let us introduce the constant

$$
\bar{E} \doteq \max _{i<j} E_{1}^{i j} \leqslant \frac{1}{4 c}\left(1+\frac{c}{2}\right)^{2}
$$

Summing up all terms in (3.16) with $j>i$, we obtain the estimate

$$
\begin{aligned}
\frac{\mathrm{d}}{\mathrm{d} t} \mathcal{Q}\left(z^{-}, z^{+}\right) & \leqslant-\frac{1}{C_{0}}\|s(t)\|_{L^{1}}+4(n-1) \bar{E} \mathcal{V}\left(z^{-}, z^{+}\right)\|s(t)\|_{L^{1}} \\
& =-\left(1-2 n C_{0} \bar{E} \mathcal{V}\left(z^{-}, z^{+}\right)\right) \frac{1}{C_{0}} \frac{\mathrm{d}}{\mathrm{d} t} \mathcal{V}\left(z^{-}, z^{+}\right) .
\end{aligned}
$$

Indeed,

$$
\frac{\mathrm{d}}{\mathrm{d} t} \mathcal{V}\left(z^{-}, z^{+}\right) \leqslant 2 n\|s(t)\|_{L^{1}}
$$


By choosing a smaller constant $\delta_{0}$, we can assume that

$$
\delta_{0} \leqslant \frac{1}{4 n C_{0} \bar{E}},
$$

so that the first two points of Theorem 1 are proved with $C=2 n C_{0}$. Indeed by (3.8), (3.18) and (3.19), it follows that the solution $\left(z^{-}, z^{+}\right)$remains in $K_{1}$ for all $t \geqslant 0$, and hence all estimates (3.9), (3.10) are valid.

To prove the Lipschitz continuous dependence with respect to the initial data, as in $[1,6]$ we consider the variational equation satisfied by an infinitesimal perturbation $\left(h^{-}, h^{+}\right)$:

$$
\left\{\begin{array}{l}
h_{t}^{-}-h_{x}^{-}=-\frac{A(u)}{2}\left(h^{+}+h^{-}\right)+\frac{h^{+}}{2}-\frac{h^{-}}{2}, \\
h_{t}^{+}+h_{x}^{+}=\frac{A(u)}{2}\left(h^{+}+h^{-}\right)-\frac{h^{+}}{2}+\frac{h^{-}}{2} .
\end{array}\right.
$$

We now project the perturbation $\left(h^{-}, h^{+}\right)$in components along the eigenvectors $r_{i}(u)$ :

$$
\begin{gathered}
h^{ \pm}=\sum_{j} h^{j \pm} r_{j}(u), \\
h_{t}^{ \pm}=\sum_{j} h_{t}^{j \pm} r_{j}(u)+\sum_{j} h^{j \pm} u_{t} \bullet r_{j} \\
=\sum_{j} h_{t}^{j \pm} r_{j}-\sum_{j k} h^{j \pm}\left(\frac{f^{k+}-f^{k-}}{2}\right) r_{k} \bullet r_{j}, \\
h_{x}^{ \pm}=\sum_{j} h_{x}^{j \pm} r_{j}+\sum_{j k} h^{j \pm}\left(\frac{f^{k+}+f^{k-}}{2}\right) r_{k} \bullet r_{j} .
\end{gathered}
$$

Each component $h^{i \pm}$ thus satisfies the equations

$$
\left\{\begin{aligned}
h_{t}^{i-}-h_{x}^{i-}= & -\left(\frac{1+\lambda_{i}(u)}{2}\right) h^{i-}+\left(\frac{1-\lambda_{i}(u)}{2}\right) h^{i+} \\
& +\sum_{k \neq j}\left\langle l^{i}, r_{k} \bullet r_{j}\right\rangle h^{j-} f^{k+}, \\
h_{t}^{i+}-h_{x}^{i+}= & \left(\frac{1+\lambda_{i}(u)}{2}\right) h^{i-}-\left(\frac{1-\lambda_{i}(u)}{2}\right) h^{i+} \\
& -\sum_{k \neq j}\left\langle l^{i}, r_{k} \bullet r_{j}\right\rangle h^{j+} f^{k-} .
\end{aligned}\right.
$$

The analysis is now very similar to the one above. Consider the functional

$$
\begin{aligned}
\mathcal{D}(z, h) \doteq & \sum_{j>i}\left\{\iint_{x<y}\left(E_{0}^{i j}+E_{1}^{i j}\right)\left\langle l_{0},\left|h^{j}(t, x)\right|\right\rangle\left\langle l_{0},\left|f^{i}(t, y)\right|\right\rangle \mathrm{d} x \mathrm{~d} y\right. \\
& +\iint_{x<y}\left(E_{0}^{i j}+E_{1}^{i j}\right)\left\langle l_{0},\left|f^{j}(t, x)\right|\right\rangle\left\langle l_{0},\left|h^{i}(t, y)\right|\right\rangle \mathrm{d} x \mathrm{~d} y
\end{aligned}
$$




$$
\begin{aligned}
& +\iint_{x>y}\left(\frac{E_{0}^{i j}}{p_{+}^{i j}\left(p_{-}^{i j}\right)^{\prime}}+\frac{E_{1}^{i j}}{p_{-}^{i j}\left(p_{+}^{i j}\right)^{\prime}}\right)\left\langle l_{1}^{i j},\left|h^{j}(t, x)\right|\right\rangle \\
& \times\left\langle\left(l_{1}^{i j}\right)^{\prime},\left|f^{i}(t, y)\right|\right\rangle \mathrm{e}^{-\alpha^{i j}(x-y)} \mathrm{d} x \mathrm{~d} y \\
& +\iint_{x>y}\left(\frac{E_{0}^{i j}}{p_{+}^{i j}\left(p_{-}^{i j}\right)^{\prime}}+\frac{E_{1}^{i j}}{p_{-}^{i j}\left(p_{+}^{i j}\right)^{\prime}}\right)\left\langle l_{1}^{i j},\left|f^{j}(t, x)\right|\right\rangle \\
& \left.\times\left\langle\left(l_{1}^{i j}\right)^{\prime},\left|h^{i}(t, y)\right|\right\rangle \mathrm{e}^{-\alpha^{i j}(x-y)} \mathrm{d} x \mathrm{~d} y\right\} \\
& \leqslant 2 \bar{E}\|h\|_{L^{1}} \mathcal{V}\left(z^{-}, z^{+}\right) .
\end{aligned}
$$

Its time derivative satisfies

$$
\begin{aligned}
\frac{\mathrm{d}}{\mathrm{d} t} \mathcal{D}(z, h) & \leqslant-\frac{1}{n C_{0}} \frac{\mathrm{d}}{\mathrm{d} t}\|h(t)\|_{L^{1}}+2 \bar{E}\|h(t)\|_{L^{1}} \frac{\mathrm{d}}{\mathrm{d} t} \mathcal{V}\left(z^{-}, z^{+}\right)+2 \bar{E} \mathcal{V}\left(z^{-}, z^{+}\right) \frac{\mathrm{d}}{\mathrm{d} t}\|h(t)\|_{L^{1}} \\
& \leqslant-\frac{1}{2 n C_{0}} \frac{\mathrm{d}}{\mathrm{d} t}\|h(t)\|_{L^{1}}+\|h(t)\|_{L^{1}} \frac{\mathrm{d}}{\mathrm{d} t} \mathcal{Q}\left(z^{-}, z^{+}\right)
\end{aligned}
$$

With easy computation one concludes

$$
\begin{aligned}
\|h(t)\|_{L^{1}} & \leqslant\|h(0)\|_{L^{1}}+2 n C_{0} \int_{0}^{t} \frac{\mathrm{d}}{\mathrm{d} s} \mathcal{D}(z, h) \exp \left\{2 n C_{0}(\mathcal{Q}(t)-\mathcal{Q}(s))\right\} \mathrm{d} s \\
& \leqslant\|h(0)\|_{L^{1}}+2 n C_{0} \mathcal{D}(z(0), h(0)) \leqslant 2\|h(0)\|_{L^{1}} .
\end{aligned}
$$

This holds for any infinitesimal perturbation. Consider a smaller domain $\mathcal{D}^{\prime} \subseteq \mathcal{D}$, such that given now two initial data $\left(z_{0}^{-}, z_{0}^{+}\right),\left(z_{1}^{-}, z_{1}^{+}\right) \in \mathcal{D}^{\prime}$, we construct the smooth path $\zeta$

$$
\theta \rightarrow \zeta(\theta)=(1-\theta)\left(z_{0}^{-}, z_{0}^{+}\right)+\theta\left(z_{1}^{-}, z_{1}^{+}\right) \in \mathcal{D}, \quad \theta \in[0,1] .
$$

If $\mathcal{S}$ denotes the semigroup generated by (1.4), one has by (3.23)

$$
\int_{0}^{1}\left\|\frac{\mathrm{d} \mathcal{S}_{t} \zeta(\theta)}{\mathrm{d} \theta}\right\|_{L^{1}} \mathrm{~d} \theta \leqslant 2 \int_{0}^{1}\|\zeta(\theta)\|_{L^{1}} \mathrm{~d} \theta, \quad \text { for all } t \in \mathbb{R}^{+} .
$$

Finally

$$
\begin{aligned}
\left\|\mathcal{S}_{t}\left(z_{0}^{-}, z_{0}^{+}\right)-\mathcal{S}_{t}\left(z_{1}^{-}, z_{1}^{+}\right)\right\|_{L^{1}} & \leqslant\left\{\int_{0}^{1}\left\|\frac{\mathrm{d} \mathcal{S}_{t} \zeta(\theta)}{\mathrm{d} \theta}\right\|_{L^{1}} \mathrm{~d} \theta\right\} \leqslant 2 \int_{0}^{1}\|\zeta(\theta)\|_{L^{1}} \mathrm{~d} \theta \\
& =2\left\|\left(z_{0}^{-}, z_{0}^{+}\right)-\left(z_{1}^{-}, z_{1}^{+}\right)\right\|_{L^{1}} .
\end{aligned}
$$

This concludes the proof of Theorem 1 , since the continuous dependence with respect to time follows from (1.4) and the fact that the solution $\left(z^{-}(t), z^{+}(t)\right)$ takes values in a compact set of $\mathbb{R}^{2 n}$.

Remark 3.2. - Since all propagation speeds are contained in the interval $[-1,1]$, a similar argument shows that 
S. BIANCHINI / Ann. Inst. Henri Poincaré Anal. nonlinear 18 (2001) 19-42

$$
\begin{aligned}
& \int_{a}^{b}\left|\left(z^{-}(t, x), z^{+}(t, x)\right)-\left(\tilde{z}^{-}(t, x), \tilde{z}^{+}(t, x)\right)\right| \mathrm{d} x \\
& \quad \leqslant L \int_{a-t}^{b+t}\left|\left(z^{-}(0, x), z^{+}(0, x)\right)-\left(\tilde{z}^{-}(0, x), \tilde{z}^{+}(0, x)\right)\right| \mathrm{d} x .
\end{aligned}
$$

\section{Proof of Theorem 2}

In this section we give a proof of Theorem 2, retracing the arguments in [6]. For each given $\varepsilon>0$, let $\mathcal{S}^{\varepsilon}$ the continuous semigroup generated by $\left(1.2^{\prime}\right)$. According to Theorem 1 , this defines a continuous semigroup. However but as $\varepsilon \rightarrow 0$, dependence on time is not uniformly continuous.

Define

$$
Z(t) \doteq \sum_{i=1}^{n} \int_{\mathbb{R}}\left|\left\langle l_{i}(u(-\infty)), v(t, x)-F(u(t, x))\right\rangle\right| \mathrm{d} x
$$

With easy computations, since the total variation of $u$ is bounded, we have

$$
\dot{Z}(t)=\mathcal{O}(1)\left\{-\frac{Z(t)}{\varepsilon}+\text { Tot.Var. }(u(t))\right\} .
$$

Therefore

$$
Z(t)=\mathcal{O}(1)\left\{Z_{0} \mathrm{e}^{-t / \varepsilon}+\varepsilon\right\} .
$$

By (1.2) this implies that the semigroup $\mathcal{S}^{\varepsilon}$ is uniformly Lipschitz continuous if $t \geqslant$ $\varepsilon \log \varepsilon$. Moreover by (1.2) one has

$$
\left\|u(t)-u_{0}\right\|_{L^{1}} \leqslant t \cdot \operatorname{Tot} . \operatorname{Var} .(v) .
$$

By a standard compactness argument, this proves the convergence to a unique Lipschitz semigroup for all $t \geqslant 0$, which can be characterized as the unique entropic solution to (1.1) constructed by wave front tracking (see [1,6]). Moreover (4.2) implies $v(t)=F(u(t))$ for all $t>0$. Formula (1.11) follows easily from (1.8).

Remark 4.1. - One sees that the discontinuous behavior of $\mathcal{S}^{\varepsilon}$ is due to the exponentially fast decay of $v(t)$ to an $\varepsilon$-neighborhood of $F(u(t))$ in the $L^{1}$ norm, where the long time dynamics takes place.

\section{Acknowledgement}

This research was partially supported by the European TMR Network on Hyperbolic Conservation Laws ERBFMRXCT960033. 


\section{REFERENCES}

[1] Bianchini S., Bressan A., BV solutions for a class of viscous hyperbolic systems, Indiana Univ. Math. J. (to appear).

[2] Berenstein C.A., Gay R., Complex Variables. An Introduction, Springer, New York, 1991.

[3] Bressan A., Hyperbolic Systems of Conservation Laws. The One Dimensional Cauchy Problem, Oxford University Press (to appear).

[4] Bressan A., The unique limit of the Glimm scheme, Arch. Rational Mech. Anal. 130 (1995) 205-230.

[5] Bressan A., Liu T.P., Yang T., $L^{1}$ stability estimates for $n \times n$ conservation laws, Arch. Rational Mech. Anal. 149 (1) (1999) 1-22.

[6] Bressan A., Shen W., BV estimates for multicomponent chromatography with relaxation, Discrete Cont. Dynamical Systems 6 (1) (2000) 21-38.

[7] Chen G.Q., Liu T.P., Zero relaxation and dissipation limits for systems of conservation laws, Comm. Pure Appl. Math. 43 (1993) 755-781.

[8] Jin S., Xin Z.P., The relaxation schemes for systems of conservation laws in arbitrary space dimensions, Comm. Pure Appl. Math. 48 (1995) 235-277.

[9] Liu T.P., Hyperbolic conservation laws with relaxation, Commun. Math. Phys. 108 (1987) 153-175.

[10] Natalini R., Convergence to equilibrium for the relaxation approximations of conservation laws, Comm. Pure Appl. Math. 49 (1998) 795-823.

[11] Natalini R., Recent results on hyperbolic relaxation problems, in: Freistühler H. (Ed.), Analysis of Systems of Conservation Laws, Chapman \& Hall/CRC, 1998, pp. 128-198. 